\title{
Construction of a Dimethyl Sulfoxide Sensor Based on Dimethyl Sulfoxide Reductase Immobilized on a Au Film Electrode
}

\author{
Hiroki Yonehara, $*$ Shin-ichiro FuJII, ${ }^{* *}$ Kiichi Sato, ${ }^{*}$ Mitsuru Abo, ${ }^{* \dagger}$ and Etsuro Yoshimura* \\ *Department of Applied Biological Chemistry, Graduate School of Agricultural and Life Sciences, \\ The University of Tokyo, Yayoi 1-1-1, Bunkyo, Tokyo 113-8657, Japan \\ **Institute for Biological Resources and Functions, National Institute of Advanced Industrial Science and \\ Technology (AIST), Central 6, 1-1-1 Higashi, Tsukuba, Ibaraki 305-8566, Japan
}

\begin{abstract}
A novel dimethyl sulfoxide (DMSO) sensor using DMSO reductase and film electrodes was constructed. The $\mathrm{Au}$ and $\mathrm{Ag}$ electrodes were fabricated on slide glass by vacuum deposition and the application of a photolithographic technique. The micro-chamber $(4 \times 50 \times 1 \mathrm{~mm}$, volume $200 \mu \mathrm{l})$ was fabricated on a poly(dimethylsiloxane) (PDMS) polymer. The Pt electrode was implanted in a PDMS polymer. DMSO reductase was immobilized on a Au film electrode with bovine serum albumin (BSA)-glutaraldehyde. This sensor could determine DMSO in an unpurged aqueous solution with glucose oxidase (GOD) and catalase (CAT) for oxygen removal. The DMSO sensor showed a linear response within $1 \mathrm{mM}$ DMSO with a correlation coefficient of 0.999 . The detection limit was $200 \mu \mathrm{M}(3 \sigma)$, and the sensitivity was $23.8 \mathrm{~mA}$ $\mathrm{M}^{-1} \mathrm{~cm}^{-2}$. The relative standard deviations at each concentration were within $3.6 \%$.
\end{abstract}

(Received September 8, 2006; Accepted November 13, 2006; Published January 10, 2007)

\section{Introduction}

Dimethyl sulfoxide (DMSO) is known as an organic solvent, but it is also a natural product. Recently, DMSO has been used as a washing solvent in the semiconductor industry, and it is sometimes contained in waste water at the millimolar level. ${ }^{1}$ DMSO has also been found in foods and beverages, such as wine, coffee, and tea, at the micromolar level.2,3 Dimethyl sulfide (DMS), the reductive product of DMSO, is unfavorable for foods or beverages because it has an offensive smell. DMSO was measured as a DMS potential source in their quality control.

Simple analytical methods for aqueous DMSO samples in various concentration ranges are desirable for the quality control of foods or beverages and environmental monitoring. However, such methods are limited because DMSO has no characteristic absorption spectrum for specific detection and is highly soluble in water, and its extraction from water is difficult. These characteristics restrict derivatization methods for DMSO. Conventionally, a purge and trap-GC method was used. ${ }^{4}$ In this method, DMSO was reduced to DMS, and gaseous DMS was concentrated on an absorbent, and then injected to a GC column by rapid heating of the absorbent. This method is highly sensitive, but the analytical procedure was complicated. In addition, it is difficult to reproduce the analytical system and measurements. ${ }^{5} \quad$ On the other hand, an electrochemical enzymatic biosensor is a suitable analytical method for aqueous DMSO samples because there is no need for the separation of DMSO and water. In addition, the electrochemical enzymatic biosensor makes the analytical instruments compact.

DMSO reductase is the terminal enzyme in DMSO respiration

$\doteqdot$ To whom correspondence should be addressed.

E-mail: abo@mail.ecc.u-tokyo.ac.jp and catalyzes the reduction of DMSO to DMS. ${ }^{6}$ Some reductive enzymes, such as molybdenum enzymes, ${ }^{7}$ are sensitive to oxygen, but DMSO reductase from Rhodobacter sphaeroides $\mathrm{f}$. sp. denitrificans is stable under the aerobic condition and it was the first enzyme of the DMSO reductase family to be characterized by X-ray crystallography. ${ }^{8}$ DMSO reductase from this strain was used for the DMSO sensor because of its stability.

Direct electron transfer to the molybdenum cofactor in DMSO reductase was observed by square wave voltammetry, ${ }^{9}$ but its rate was insufficient for an amperometric sensor. Methyl viologen (MV) was used as a mediator because of its fast electron transfer between the electrode and the enzyme. The overall electro-enzymatic reaction is as follows:

$$
\mathrm{DMSO}+2 \mathrm{H}^{+}+2 \mathrm{MV}^{+\bullet}=\mathrm{DMS}+\mathrm{H}_{2} \mathrm{O}+2 \mathrm{MV}^{++},
$$

where $\mathrm{MV}^{+}$and $\mathrm{MV}^{++}$represent the radical cation form and the dication form of MV, respectively.

In general, reductive electrochemical biosensors suffer from oxygen interference. The dissolved oxygen in aqueous samples is usually removed by nitrogen or argon bubbling before electrochemical measurements, but the obtained anaerobic level is affected by various experimental conditions, such as the flow rate of the bubbling gas and the headspace volume of the sample vials. The glucose oxidase (GOD)-catalase (CAT) system was used in a nitric oxide (NO) reductase assay for removing oxygen. ${ }^{10,11}$ Molecular oxygen is reduced into hydrogen peroxide by glucose oxidase, and is further transformed into water and a half mole of oxygen by catalase. This enzymatic system can achieve and strictly sustain anaerobic conditions because NO reacts readily with molecular oxygen. This oxygen-removal procedure was applied to the analysis of aqueous DMSO samples. 


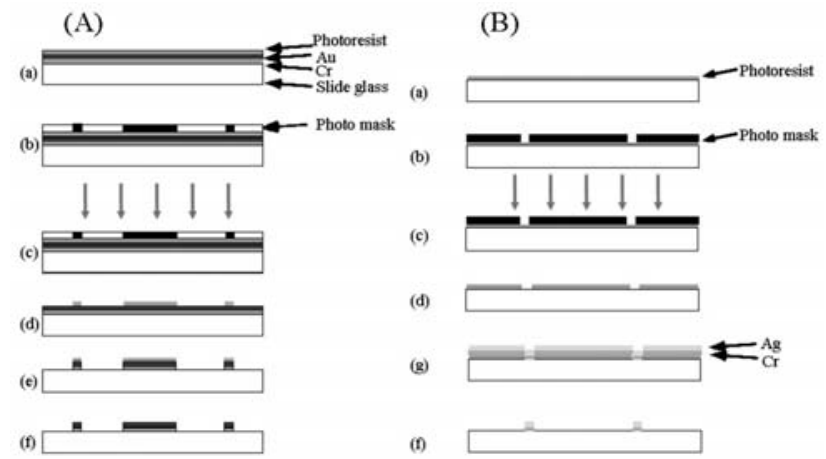

Fig. 1 Fabrication process of a Au film electrode (A) and a Ag film electrode (B) onto slide glass. A, Etching method; B, lift-off method; a, spreading photoresist; b, covering the photomask; c, UV irradiation; d, removing resist pattern; e, deposition $\mathrm{Cr}$ and $\mathrm{Au}$; $\mathrm{f}$, removing photoresist; g, deposition $\mathrm{Cr}$ and $\mathrm{Ag}$.

The use of film electrodes makes the analytical device more compact, and the micro-chamber is suitable for a reductive electrochemical reaction because of reduction of the oxygen interference. For these reasons, we tried to construct a DMSO sensor with film electrodes, which is useful for in situ environmental monitoring or the quality control of foods or beverages. In this paper, the basic characteristics and the fabrication of the DMSO sensor are described.

\section{Experimental}

\section{Chemicals and apparatus}

A positive photoresist (OFPR-800; $34 \mathrm{cP}$ ) and developer (NMD-3) were obtained from Tokyo Ohka Kogyo (Kanagawa, Japan). The gold etching solution consisted of iodine and ammonium iodide $(1.2 \mathrm{~g}$ iodine and $3.0 \mathrm{~g}$ ammonium iodide $/ 100 \mathrm{ml})$. The chromium etching solution contained ammonium cerium nitrate $(13.3 \%, \mathrm{w} / \mathrm{v})$. The PDMS elastomer (SILPOT $184 \mathrm{~W} / \mathrm{C}$ ) was obtained from Dow Corning Toray (Tokyo, Japan). A spin-coater (SC308; Union Optical Co.) was used for photolithography. A UV lamp (HB-25105AA) was obtained from Ushio, Inc. (Kanagawa, Japan). Glucose oxidase, catalase, BSA, glucose, and a 25\% glutaraldehyde solution (GA solution) were obtained from Wako Pure Chemical Industries. Methyl viologen was obtained from Tokyo Chemical Industry, Co. Chloridization silver ink was purchased from BAS, Inc.

\section{Enzyme preparation}

DMSO reductase was purified from Rhodobacter sphaeroides forma specialis denitrificans by Satoh's procedure. ${ }^{12}$

\section{Electrode fabrication}

Film electrodes were fabricated on the slide glass by vacuum evaporation and applying the photolithographic technique. ${ }^{13}$ The $\mathrm{Au}$ working electrode was fabricated with an etching method, and the $\mathrm{Ag} / \mathrm{AgCl}$ reference electrode was fabricated with a lift-off method.

At first, the working and counter electrodes were fabricated in the same manner, but the counter electrode was easily peeled off during the electrochemical reaction (data not shown). Platinum (Pt) wire was used as a counter electrode, which was implanted in the PDMS polymer.

The fabrication steps are illustrated in Fig. 1. First, $\mathrm{Cr}$ and $\mathrm{Au}$ were deposited on a slide glass. The positive photoresist

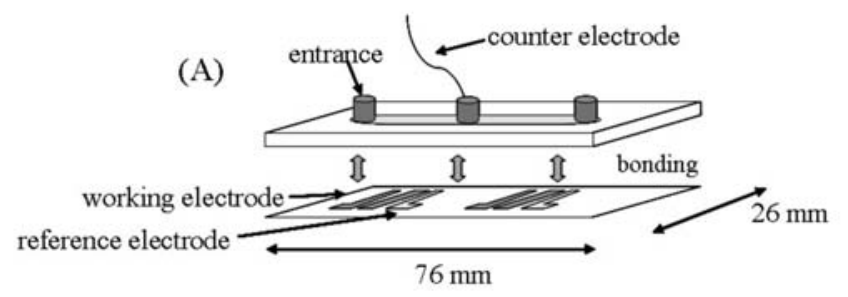

(B)

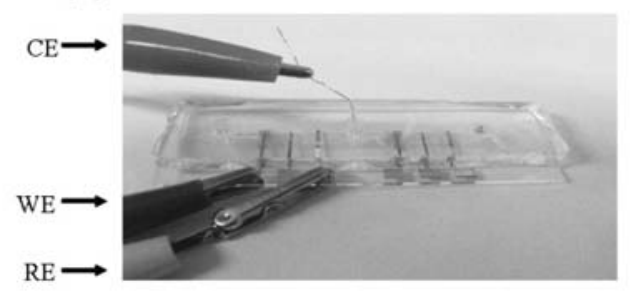

Fig. 2 Schematic structure (A) and photograph (B) of the DMSO sensor. Film electrodes were formed on slide glass, and Pt wire was implanted in PDMS. A micro-chamber was molded in PDMS.

(OFPR-800, $34 \mathrm{cP}$ ) was spin-coated with a spin-coater onto a slide glass at $500 \mathrm{rpm}$ for $3 \mathrm{~s}$ and then at $2000 \mathrm{rpm}$ for $20 \mathrm{~s}$. The glass coated with the photoresist was heated at $90^{\circ} \mathrm{C}$ for 30 min on a hotplate. Next, a photo mask was set on the slide glass, and the glass was exposed to UV light for $1 \mathrm{~min}$. The slide glass was immersed in NMD-3 for 2 min and then rinsed with distilled water. Similarly, the slide glass was immersed in the gold etching solution and chrome etching solution for $1 \mathrm{~min}$. Finally, the slide glass was washed with acetone to remove the photoresist. Next, the silver electrode was fabricated on the slide glass. As shown in Fig. 1B, the pattern of the Au film electrode was covered with a photoresist, and the pattern of the $\mathrm{Ag}$ electrode was formed using photolithography. After washing the photoresist with NMD-3, Cr and Ag were deposited on the slide glass by vacuum evaporation, and the slide glass was then washed with acetone. The $\mathrm{Ag} / \mathrm{AgCl}$ electrode was fabricated by electrochemical chloridization or by spreading chloridization silver ink on the silver electrode.

\section{Casting of PDMS}

PDMS was used as the base material because of its ease of fabrication. A 10:1 combination of a PDMS elastomer and a curing agent was mixed and then thoroughly degassed. The polymer mixture was cast into a mold with silicon tubes, which were laid on the micro-chamber and left for $20 \mathrm{~h}$ at room temperature. The PDMS replica was then peeled from the mold, which was made of a plastic film on slide glass. The PDMS replica could be bonded on the slide glass on which the film electrodes were fabricated (Fig. 2A).

The size of the micro-chamber was $4 \times 50 \times 1 \mathrm{~mm}$, and the volume was $200 \mu \mathrm{L}$. The connectors were made of a silicon tube, which make it easy to inject the solution in the microchamber by pipetting.

\section{Enzyme immobilization}

Before enzyme immobilization, the Au film electrode was washed with ethanol for $5 \mathrm{~min}$ using an ultrasonic cleaning apparatus. Enzyme immobilization on the Au film electrode took place by placing $5 \mu \mathrm{L}$ of the enzyme solution $(10 \%$ BSA:DMSO-R $(320 \mathrm{U} / \mathrm{ml}): 5 \%$ GA solution $=4: 2: 1)$ on the Au film electrode and air-drying for $30 \mathrm{~min}$. 


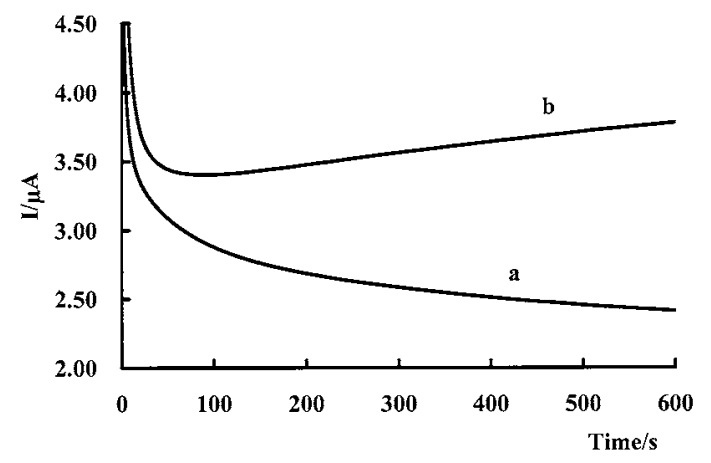

Fig. 3 Background current with and without the glucose oxidasecatalase system. Typical amperometric signals with (a) and without (b) glucose, glucose oxidase and catalase for removal of the dissolved oxygen. The applied potential was $-750 \mathrm{mV}$ vs. $\mathrm{Ag} / \mathrm{AgCl}$. The concentration of MV was $1 \mathrm{mM}$ in $100 \mathrm{mM}$ potassium phosphate buffer (pH 6.5).

\section{Electrochemical measurement}

Cyclic voltammetry and chronoamperometry were performed using an electrochemical analyzer (ALS 832A, BAS). The three-electrode system consisted of a DMSO-R immobilized film gold electrode as a working electrode, a platinum wire as a counter electrode, and a $\mathrm{Ag} / \mathrm{AgCl}$ electrode as a reference electrode.

Amperometric measurements were carried out at room temperature in a $100 \mathrm{mM}$ potassium phosphate buffer $(\mathrm{pH} 6.5)$ containing glucose oxidase $(8 \mathrm{U} / \mathrm{ml})$, catalase $(230 \mathrm{U} / \mathrm{ml})$, and glucose $(100 \mathrm{mM})$ for removal of dissolved oxygen. ${ }^{14}$ Sample solutions were deoxygenated for $30 \mathrm{~min}$ before electrochemical measurements. A bare $\mathrm{Au}$ film electrode was used for the oxygen interference experiment and the DMSO reductase immobilized $\mathrm{Au}$ film electrode was used for the DMSO measurement.

\section{Results and Discussion}

A microchip was fabricated as shown in Fig. 2(A, B), which consisted of film electrodes on slide glass, a micro-chamber, and three connectors, one of which was used as a Pt wire electrode. DMSO reductase was immobilized on a $\mathrm{Au}$ film electrode with BSA-glutaraldehyde.

First, the interference of the dissolved oxygen was examined, using a bare Au film electrode. The cyclic voltammetry of MV in phosphate buffer ( $\mathrm{pH}$ 6.5) showed a broad and irreversible peak derived from dissolved oxygen in addition to the reduction peak of $\mathrm{MV}^{++}$(data not shown). The background signals of MV solutions with and without the GOD-CAT system were measured. As shown in Fig. 3, the amperometric signal with the GOD-CAT system significantly decreased, and showed a constant value. The amplitude of this signal was reproducible and the relative standard deviation (RSD) of the signal, $10 \mathrm{~min}$ after the potential was applied, was $2.1 \%(n=3)$. These results showed that the GOD-CAT system offered sufficient and reproducible anaerobic conditions, although PDMS is a gaspermiable membrane.

The amperometric signal of the DMSO sensor is shown in Fig. 4. Before a measurement, standard solutions of DMSO in various concentrations were deoxygenated using the GOD-CAT system to eliminate the background current derived from the dissolved oxygen. They were then injected into the chamber,

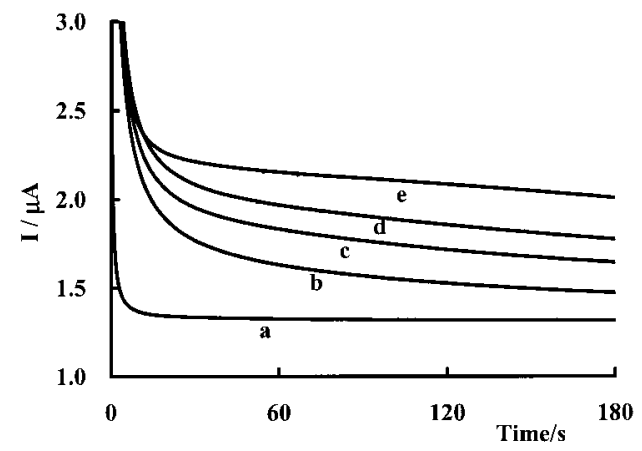

Fig. 4 Typical amperometric signals of a DMSO sensor. The DMSO concentrations were a, $0 ; \mathrm{b}, 0.25 ; \mathrm{c}, 0.50 ; \mathrm{d}, 0.75 ; \mathrm{e}, 1.00$ $\mathrm{mM}$. DMSO solutions in various concentrations in a $100 \mathrm{mM}$ potassium phosphate buffer ( $\mathrm{pH}$ 6.5) contained glucose, glucose oxidase, and catalase for removing soluble oxygen. The other conditions are the same as in Fig. 3.

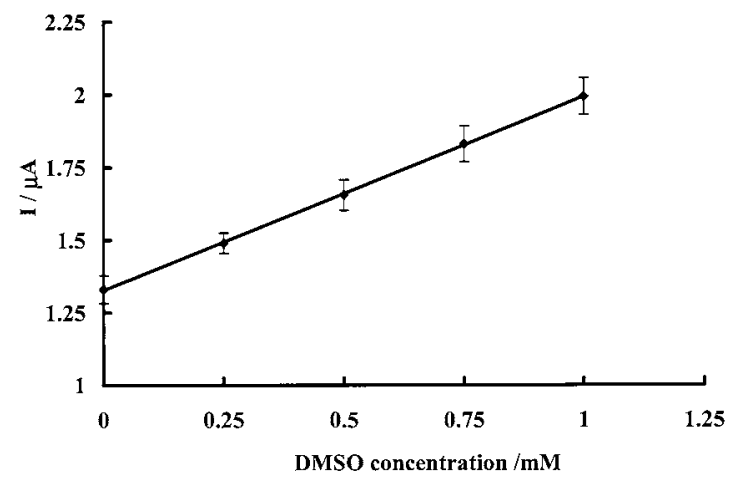

Fig. 5 Calibration curve for DMSO. The current responses at $180 \mathrm{~s}$ are plotted. The values are the average of four measurements at each concentration.

and the amperometric signals were recorded. The applied potential was fixed at the peak potential of $\mathrm{MV}^{+\bullet} / \mathrm{MV}^{++}$. The signals were increased with the concentration of DMSO.

Amperometric signals $180 \mathrm{~s}$ after applying a potential were recorded; the calibration curve for DMSO is shown in Fig. 5. The signals increased linearly, corresponding to the concentration of DMSO within $1 \mathrm{mM}$, with a correlation coefficient of 0.999 . The relative standard deviations (RSD) at each concentration were within $3.6 \%$. The lower quantification limit was $200 \mu \mathrm{M}$ $(3 \sigma)$, and the sensitivity was $23.8\left(\mathrm{~mA} \mathrm{M}^{-1} \mathrm{~cm}^{-2}\right)$.

Next, the stability of this sensor was examined. As shown in Fig. 6, about one half of the response was maintained within 6 days, but, after 8 days, the response was significantly reduced. DMSO reductase from $R$. sphaeroides is a stable enzyme, and ca. $20 \%$ of its initial activity was maintained for 18 days by BSA-glutaraldehyde immobilization on a glassy carbon electrode; ${ }^{15}$ therefore, this significant loss of response is mainly due to enzyme film peeling. The calibration range was narrower as the signal response decreased, but a sufficient response was maintained for a couple of days, which is sufficient for disposable use.

\section{Conclusions}

A novel DMSO sensor using DMSO reductase and film 


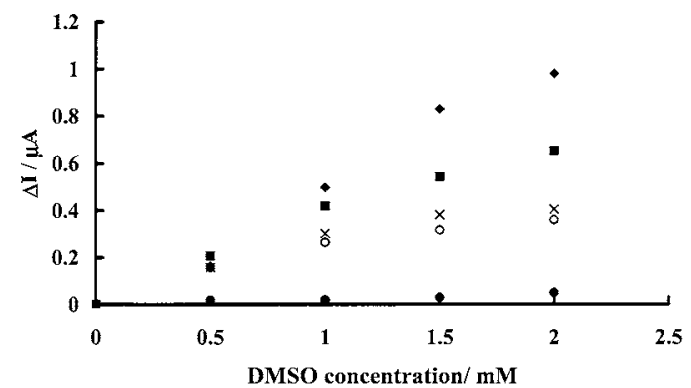

Fig. 6 Stability of the DMSO sensor. The current responses after subtracting the background obtained by the DMSO sensor $0(\diamond), 2$ $(\square), 4(\times), 6(0)$, and $8(\bullet)$ days after the enzyme had been immobilized. The other conditions are the same as in Fig. 3.

electrodes was constructed. This sensor is compact, and the sample volume was considerably reduced to $200 \mu \mathrm{L}$ relative to that of the conventional batch sensor. This sensor showed a linear response within $1 \mathrm{mM}$ DMSO and a good repeatability $(\mathrm{RSD}<3.6 \%)$. The lower detection limit was $200 \mu \mathrm{M}(3 \sigma)$; this concentration range covers wastewater samples and some food and beverage samples.

This paper also demonstrates that the GOD-CAT oxygen removal system effectively worked, and offers sufficient and reproducible anaerobic conditions, which are required for a reductive electrochemical sensor. This system is a better oxygen removal procedure than the purging method, and offers anaerobic conditions in the PDMS, which is a widely used mateial in microfabrication.

This sensor is still in its preliminary form, but it has the potential for simple and in situ analysis of aqueous DMSO samples.

\section{Acknowledgements}

This study was supported by a Grant-in-Aid for Scientific Research (No. 18780084) from the Ministry of Education, Science, Sports and Culture of Japan.

\section{References}

1. S. Park, T. Yoon, J. Bae, H. Seo, and H. Park, Process Biochem., 2001, 36, 579.

2. S. J. Mora, P. Lee, D. Shooter, and R. Eschenbruch, Am. J. Enol. Vitic, 1993, 44, 327.

3. T. W. Pearson, H. J. Dawson, and H. B. Lackey, J. Agric. Food Chem., 1981, 29, 1089.

4. R. Simo, G. Malin, and P. S. Liss, Anal. Chem., 1998, 70, 4864.

5. K. Ui, M. Abo, A. Okubo, and S. Yamazaki, Anal. Sci., 2004, 20, 223

6. T. Satoh, Y. Hoshino, and H. Kitamura, Arch. Microbiol., 1976, 108, 265.

7. R. Hille, Chem. Rev., 1996, 96, 2757.

8. H. Schindelin, C. Kisker, J. Hilton, K. V. Rajagopalan, and D. C. Rees, Science, 1996, 272, 1615.

9. K. F. Aguey-Zinsou, P. V. Bernhardt, A. G. McEwan, and J. P. Ridge, J. Biol. Inorg. Chem., 2002, 7, 879.

10. T. Fujiwara and Y. Fukumori, J. Bacteriol., 1996, 178, 1866.

11. G. J. Carr and S. J. Ferguson, Biochem. J., 1990, 269, 423.

12. T. Satoh and F. N. Kurihara, J. Biochem., 1987, 102, 191.

13. S. Fujii, T. Tokuyama, M. Abo, and A. Okubo, Analyst, 2004, 129, 305.

14. H. Cheng, M. Abo, and A. Okubo, Analyst, 2003, 128, 724.

15. M. Abo, Y. Ogasawara, Y. Tanaka, A. Okubo, and S. Yamazaki, Biosens. Bioelectron., 2003, 5, 735. 